\title{
Facet-Selective Growth on Nanowires Yields Multi- Component Nanostructures and Photonic Devices
}

\section{Citation}

Kempa, Thomas J., Sun-Kyung Kim, Robert W. Day, Hong-Gyu Park, Daniel G. Nocera, and Charles M. Lieber. 2013. "Facet-Selective Growth on Nanowires Yields Multi-Component Nanostructures and Photonic Devices." Journal of the American Chemical Society 135 (49) (December 11): 18354-18357. doi:10.1021/ja411050r.

\section{Published Version}

doi:10.1021/ja411050r

\section{Permanent link}

http://nrs.harvard.edu/urn-3:HUL.InstRepos:13364826

\section{Terms of Use}

This article was downloaded from Harvard University's DASH repository, and is made available under the terms and conditions applicable to Open Access Policy Articles, as set forth at http:// nrs.harvard.edu/urn-3:HUL.InstRepos:dash.current.terms-of-use\#OAP

\section{Share Your Story}

The Harvard community has made this article openly available.

Please share how this access benefits you. Submit a story.

\section{Accessibility}




\title{
Facet-Selective Growth on Nanowires Yields Multi- Component Nanostructures and Photonic Devices
}

\author{
Thomas J. Kempa, ${ }^{\dagger}$ Sun-Kyung Kim, ${ }^{\dagger, \diamond}$ Robert W. Day, ${ }^{\dagger}$ Hong-Gyu Park, ${ }^{* \S}$ Daniel G. Nocera, ${ }^{* \dagger}$ and \\ Charles M. Lieber* ${ }^{\dagger}, \dot{\dagger}$ \\ ${ }^{\dagger}$ Department of Chemistry and Chemical Biology and ${ }^{*}$ School of Engineering and Applied Sciences, Harvard University, \\ Cambridge, Massachusetts 02138. 'Department of Applied Physics, Kyung Hee University, Gyeonggi-do, Republic of Ko- \\ rea. ${ }^{\S}$ Department of Physics, Korea University, Seoul 136-701, Republic of Korea.
}

\section{Supporting Information Placeholder}

\begin{abstract}
Enhanced synthetic control of the morphology, crystal structure, and composition of nanostructures can drive advances in nanoscale devices. Axial and radial semiconductor nanowires are examples of nanostructures with one and two structural degrees of freedom, respectively, and their synthetically tuned and modulated properties have led to advances in nanotransistor, nanophotonic, and thermoelectric devices. Similarly, developing methods that allow for synthetic control of greater than two degrees of freedom could enable new opportunities for functional nanostructures. Here we demonstrate the first regioselective nanowire shell synthesis in studies of $\mathrm{Ge}$ and $\mathrm{Si}$ growth on faceted Si nanowire surfaces. The selectively deposited Ge is crystalline and its facet position can be synthetically controlled in situ. We use this synthesis to prepare electrically-addressable nanocavities into which solution soluble species such as Au nanoparticles can be incorporated. The method furnishes multi-component nanostructures with unique photonic properties and presents a more sophisticated nanodevice platform for future applications in catalysis and photodetection.
\end{abstract}

Semiconductor nanowires (NWs) represent a diverse class of nanomaterials whose synthetically-tunable structural, electronic, and optical properties ${ }^{1-3}$ have enabled active nanodevices including high-performance field-effect transistors, ${ }^{4}$ ultra-sensitive biological probes, ${ }^{5-7}$ and solar cells and photonic devices with tunable optical spectra. ${ }^{8-12}$ NWs can be classified according to the number of degrees of freedom (DoF) they possess, which represent fundamental physical coordinates along which their structure can be manipulated. Axial and radial (core/shell) modulated NWs have 1 and 2 DoF, respectively, and have been extensively studied and characterized. ${ }^{2,13-19}$ Nevertheless, the properties of nanostructures possessing greater complexity and anisotropy have not been determined.

A nanostructure with $3 \mathrm{DoF}$ and higher can be realized by breaking the rotational symmetry of conventional radial shell growth (Figure 1A). A high-resolution scanning electron micrograph (SEM) of a faceted core/shell Si NW (Figure 1B) reveals well-defined surfaces that were previously indexed ${ }^{9}$ as $\{111\},\{011\}$, and $\{113\}$. NWs with this same morphology and set of surface facets serve as the faceted templates from which all subsequent nanostructures in this study are grown. Following chemical vapor deposition (CVD) synthesis of the SiNW templates, ${ }^{9}$ introduction of $\mathrm{GeH}_{4}$ and $\mathrm{H}_{2}$ at lower tem-
(A)

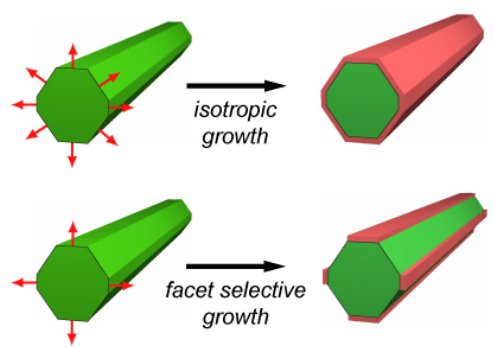

(B)

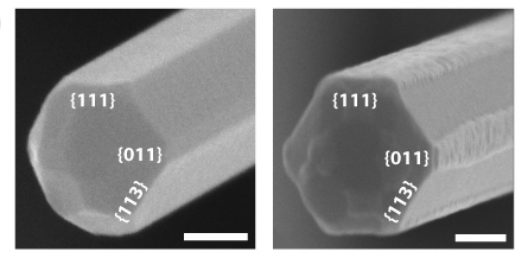

(C)

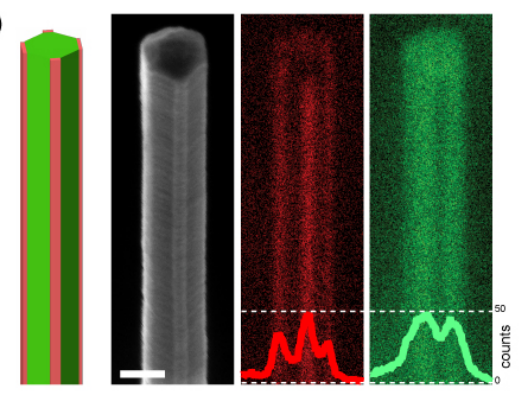

(D)

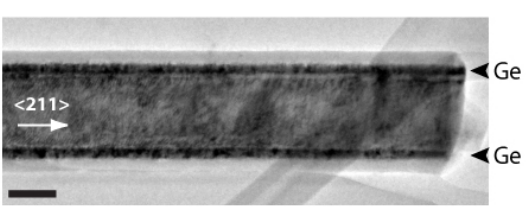

Figure 1. (A) Schematics depicting isotropic (top) versus anisotropic (bottom) growth of $\mathrm{Ge}$ (red) on a faceted $\mathrm{Si}$ template (green). (B) SEM of faceted Si template (left) and of nanostructure (right) after selective deposition of $10 \mathrm{~nm}$ of Ge (lighter contrast) on Si $\{111\}$ and $\{011\}$ surfaces. Images are oriented with $\{111\}$ surfaces on top and bottom. Scale bars, $100 \mathrm{~nm}$. (C) Schematic and SEM of a single nanostructure. Ge (red) and Si (green) EDS elemental maps of the same nanostructure and line profiles extracted from signal counts along the x-axis of the images. Images are oriented with $\{011\}$ surface facing the reader. Scale bar, $200 \mathrm{~nm}$. (D) Bright-field planview TEM of nanostructure. Scale bar, $100 \mathrm{~nm}$. 
(A)

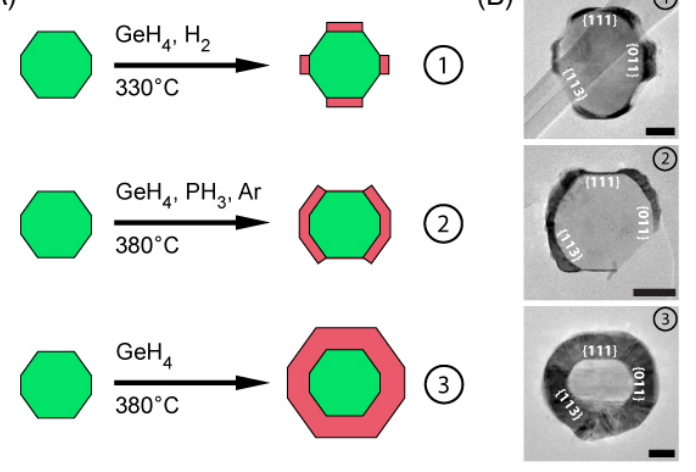

Figure 2. (A) Schematics summarizing three syntheses conducted to explore control of facet selective growth of $\mathrm{Ge}$ (red) on a faceted Si template (green). (B) Bright-field TEMs of $40 \mathrm{~nm}$ thick cross-sections of nanostructures 1-3 prepared according to the syntheses outlined in (A). Images are oriented with $\{111\}$ surfaces on top and bottom. Scale bars, $50 \mathrm{~nm}$.

perature and pressure into the same reactor (Supporting Information) yields a new product featuring selective material deposition on the $\{111\}$ and $\{011\}$ Si surface facets (Figure 1B). Energy dispersive x-ray spectroscopy (EDS) performed on the nanostructure (Figure 1C) confirms the elemental identity of the deposited material as Ge and reveals that facet selectivity is preserved along the length of the nanostructure. A planview transmission electron micrograph (TEM) of the anisotropic SiGe nanostructure (Figure 1D) reveals mesas with a uniform dark contrast corresponding to Ge. The Ge appears smooth and ordered and extends uniformly along the length of the $\{011\}$ facet to which it is bound. Notably, these results differ significantly from the disordered, island morphologies that typify Stranski-Krastanov growth of Ge on planar and nanoscale Si surfaces. ${ }^{20-23}$

We performed syntheses using different Si templates, temperatures, and gas-phase species to determine their role in growth of Si-Ge (heteroepitaxial) and $\mathrm{Si}-\mathrm{Si}$ (homoepitaxial) nanostructures with distinct anisotropies. After performing each synthesis for 5 min (Figure 2A, Figures S1 and S2), we prepared $40 \mathrm{~nm}$ thick cross-sections of the synthesized nanostructures and analyzed their morphology by bright-field TEM (Figure 2B, Figures S1 and S2).

TEM analysis of a nanostructure synthesized at $330{ }^{\circ} \mathrm{C}$ with $\mathrm{GeH}_{4}$ and $\mathrm{H}_{2}$ reveals dark contrast corresponding to Ge that has selectively grown on the $\{111\}$ and $\{011\}$ surfaces of the template (Figure 2: structure 1). No dark contrast is visible on the $\{113\}$ Si surfaces, thus reinforcing our previous contention of high selectivity for this Ge growth (Figure 1). Some variation in the Si morphology reflects both natural dispersion and TEM sample preparation (Figure S3 and Supporting Information). From TEM measurements and Ge growth time, we estimate the growth rates of $\mathrm{Ge}$ on $\{011\}$ and $\{111\}$ surfaces to be $2 \mathrm{~nm} / \mathrm{min}$ and $1 \mathrm{~nm} / \mathrm{min}$, respectively. Repeating this synthesis in the presence of the faceted Si template coated with a $3 \mathrm{~nm}$ thick amorphous $\mathrm{Si}$ layer yields a thin isotropic $\mathrm{Ge}$ shell (Figure S1). Interestingly, growth at $650{ }^{\circ} \mathrm{C}$ with $\mathrm{SiH}_{4}$ and $\mathrm{H}_{2}$ leads to selective $\mathrm{Si}$ growth on the $\{111\}$ surfaces of the Si template (Figure S2). This result is in contradistinction to the isotropic nanocrystalline $\mathrm{Si}$ shell ${ }^{24}$ observed when this synthesis is performed on an axial $\mathrm{Si} \mathrm{NW}$ without welldefined surface facets. Together, these results demonstrate that the surface properties of the template play a vital role in enabling and controlling facet selective growth.

To determine whether Ge can be selectively grown on other facets, we examined syntheses at higher temperature and with various gas-phase precursors. TEM analysis of a nanostructure synthesized at $380^{\circ} \mathrm{C}$ with $\mathrm{GeH}_{4}, \mathrm{PH}_{3}$, and Ar reveals (Figure 2 , structure 2) estimated Ge growth rates on the $\{113\},\{011\}$, and $\{111\}$ surfaces of $3.0,1.6$, and $0.3 \mathrm{~nm} / \mathrm{min}$, respectively. In structure 2, a $\times 10$ faster growth rate of $\mathrm{Ge}$ on $\{113\}$ versus $\{111\}$ is a striking reversal of the observed growth trend on these surfaces for structure $\mathbf{1}$. We determined that Ar alone enhances growth of $\mathrm{Ge}$ on $\{113\}$ versus $\{111\}$ whereas $\mathrm{PH}_{3}$ improves selectivity by suppressing Ge growth on the $\{111\}$ surface, likely through passivation of $\mathrm{Si}$ surface sites with adsorbed phosphine or phosphine-derived species. ${ }^{10,25}$ Finally, TEM analysis of a nanostructure synthesized at $380{ }^{\circ} \mathrm{C}$ with $\mathrm{GeH}_{4}$ and no other gas-phase species reveals (Figure 2: structure 3) an isotropic Ge shell and an estimated growth rate for this shell of $10 \mathrm{~nm} / \mathrm{min}$. This result confirms the importance of gas-phase species in mediating facet selective growth of Ge at higher temperatures. The yields of structures 1-3 determined from random sampling are in the range of $70-90 \%$. In summary, these results represent the first gas-phase facet selective growth of $\mathrm{Ge}$ and $\mathrm{Si}$ on Si nanowire surfaces and establish that their facet position can be synthetically controlled in situ to elaborate unique nanostructures with higher anisotropy.

To explore more complex and opto-electronically active nanostructures we encapsulated nanoscale Ge regions within a Si $p-n$ interface. The targeted architecture includes a faceted template with $p$-type and intrinsic $\mathrm{Si}$ shells, facet selective grown $\mathrm{Ge}$, and finally a conformal shell of $n$-type $\mathrm{Si}$ (Figure 3A, Supporting Information). Notably, all synthetic steps were carried out in a continuous sequence in a single reactor for this complex structure. Bright-field TEM (Figure 3B) and EDS (Figure 3C,Figure S4) elemental mapping of the nanostructure cross-section verify that Ge was selectively embedded within the nanostructure while preserving the radial Si $p-n$ junction. High-resolution TEM analysis of a region of the cross-section near the Si $\{111\}$ interface (Figure 3D) reveals several important features. First, crystal lattice fringes proceed through the intrinsic $\mathrm{Si} / \mathrm{Ge} / n$-type $\mathrm{Si}$ regions of interest and terminate at the amorphous $\mathrm{SiO}_{\mathrm{x}}$ layer passivating the nanostructure. Second, two-dimensional Fourier transforms (Figure 3D) of lattice resolved TEM images from the intrinsic Si and Ge regions show well resolved spots that are consistent with the [211] zone-axis of the cubic crystal lattice. ${ }^{26}$ Third, the sharp contrast changes visible in bright-field (Figure 3D) and darkfield TEM (Figure S4 inset) depict the abrupt interfaces formed between $\mathrm{Si}$ and $\mathrm{Ge}$. In addition, high resolution TEM of a region of the cross-section near the Si $\{113\}$ interface (Figure $3 \mathrm{E}$ ) reveals an apparently clean intrinsic $\mathrm{Si} / n$-type $\mathrm{Si}$ interface. Together, these results verify that the facet selective growth of Ge and subsequent conformal growth of $n$-type Si are crystalline and that clean, atomically-sharp interfaces can be designed and realized in these complex nanostructures.

High-resolution TEM analysis of a region of the crosssection near the Si $\{011\}$ interface (Figure $3 \mathrm{~F}$ and Figure S5) reveals several unique features. First, $\mathrm{Si}\{111\}$ lattice fringes progress across the intrinsic $\mathrm{Si} / \mathrm{Ge}$ junction and are distorted near the edge of the Ge mesa where the $\mathrm{Si}\{011\}$ and $\{113\}$ surfaces meet (Figure 3F). Second, a TEM spanning the full width of this region (Figure S5) shows a region of crystalline Ge that is $15 \mathrm{~nm}$ wide. Growth of planar Ge films on Si typi- 
(A)

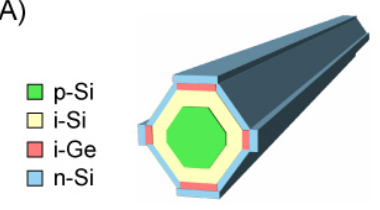

(B)

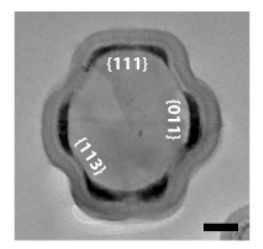

(C)

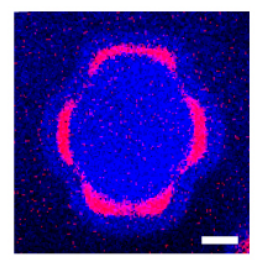

(E)

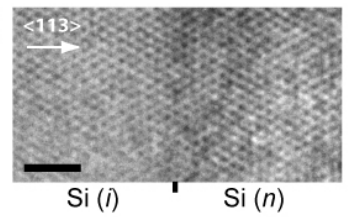

(F)

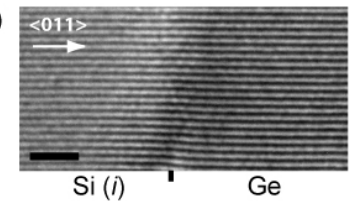

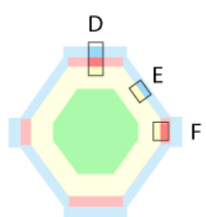

(D)

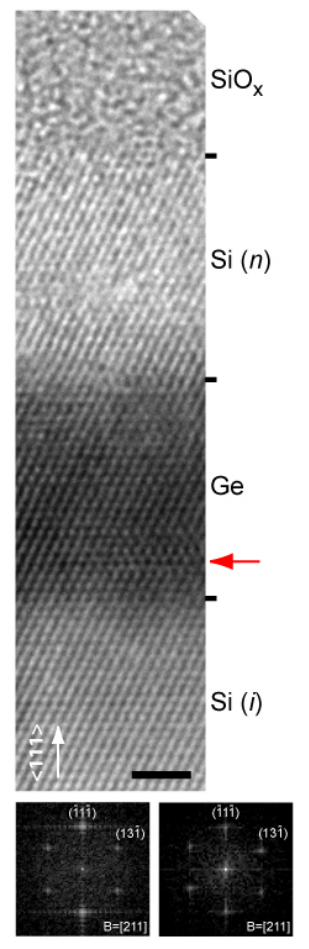

Figure 3. (A) Schematic (left) depicting a complex nanostructure with Ge regions selectively embedded within a $p$-i- $n$ junction. Schematic (right) of the nanostructure cross-section with labels D, E, F corresponding to the figure panels where these interfaces are presented in detail. (B) Bright-field TEM of a $40 \mathrm{~nm}$ thick cross-section of the active nanostructure. Image is oriented with $\{111\}$ surfaces on top and bottom. Scale bar, $50 \mathrm{~nm}$. (C) EDS elemental map of section shown in (B) revealing Ge (red) embedded in Si (blue). Scale bar, $50 \mathrm{~nm}$. (D) High-resolution TEM of the intrinsic $\mathrm{Si} / \mathrm{Ge} / n$-type $\mathrm{Si}$ region near the $\{111\}$ interface within the nanostructure. The $\{111\}$ plane lies parallel to the $\mathrm{x}-$ axis of the image. Two-dimensional FFTs of lattice-resolved TEMs of intrinsic Si (left) and Ge (right) regions. The crosssection is perpendicular to the nanostructure [211] zone axis. Red arrow indicates site of misfit dislocation. Scale bar, $2 \mathrm{~nm}$. (E) High-resolution TEM of the intrinsic $\mathrm{Si} / n$-type $\mathrm{Si}$ region near the $\{113\}$ interface within the nanostructure. The $\{113\}$ plane lies parallel to the y-axis of the image. Scale bar, $2 \mathrm{~nm}$. (F) Highresolution TEM of the intrinsic Si/Ge region near the $\{011\}$ interface within the nanostructure. The $\{011\}$ plane lies parallel to the $\mathrm{y}$-axis of the image. Scale bar, $2 \mathrm{~nm}$.

cally proceeds by the Stranski-Krastanov mechanism. ${ }^{20}$ An initial stage of epitaxial growth for thicknesses $<5 \mathrm{~nm}^{20}$ is followed by a disordered, three-dimensional (island) phase as strain energy increases due to the $4.2 \%$ Ge-Si lattice mismatch. Notably, our results demonstrate epitaxial Ge growth over greater thicknesses and suggest this is due to homogeneous relaxation of compressive $\operatorname{strain}^{27,28}$ in the $\{011\}$ plane, facilitated by the absence of crowding species on the adjacent $\{113\}$ surface. Likewise, epitaxial growth of Ge persists for

(A)

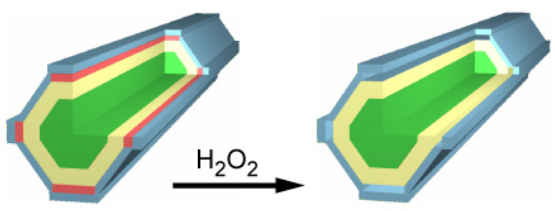

(B)
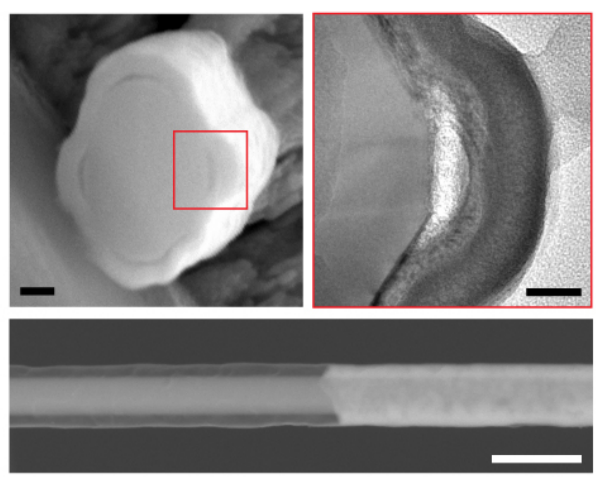

(C)
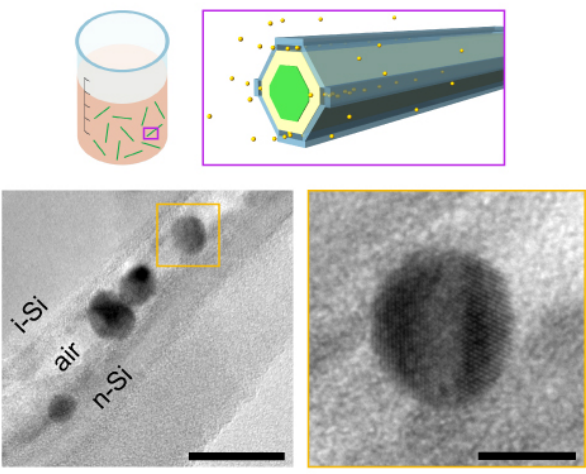

Figure 4. (A) Schematic demonstrating selective etching of $\mathrm{Ge}$ in $\mathrm{H}_{2} \mathrm{O}_{2}$ to form nanocavities. (B) SEM (left, scale bar, $50 \mathrm{~nm}$ ) of the end of an etched nanostructure; high-resolution TEM (right, scale bar, $20 \mathrm{~nm}$ ) of a cross-section near the $\{011\}$ interface. Planview SEM (bottom, scale bar, $500 \mathrm{~nm}$ ) of an etched nanostructure. (C) Schematic showing immersion of etched nanostructure in Au colloid solution. TEM image (left, scale bar, $20 \mathrm{~nm}$ ) showing $4 \mathrm{Au}$ nanoparticles infiltrated into the nanostructure cavity. High-resolution TEM image (right, scale bar, $5 \mathrm{~nm}$ ) showing magnified view of a $7 \mathrm{~nm}$ Au nanoparticle.

growth in the $<111>$ direction and shows evidence of formation of misfit dislocations ${ }^{27,28} \sim 4$ epilayers distant from the intrinsic $\mathrm{Si} / \mathrm{Ge}$ interface (Figure 3D). The properties of the localized $\mathrm{Si}-\mathrm{Ge}$ heterostructure regions and/or the role of $\mathrm{Ge}$ as a sensitizer within the nanostructure will be of future interest.

As a first step towards examining the optoelectronic properties of nanostructures with newly accessible anisotropies, we prepared a novel nanostructure with functional nanocavities. Specifically, hydrogen peroxide was used to etch Ge selectively and thus convert the nanostructure synthesized in Figure 3 to one with controlled nanocavities embedded within the $p-n$ junction (Figure 4A, Supporting Information). SEM and TEM analyses (Figure 4B) verify that well-defined nanoscale cavities were etched in place of the Ge and that they extend uniformly along the lengths of the facet, where longitudinal etch distance is a function of the $\mathrm{H}_{2} \mathrm{O}_{2}$ etch time. Next, we fabricated single nanodevices with either a 10 or $20 \mathrm{~nm}$ wide nanocavity. Experimental and simulated external quantum 
efficiency (EQE) spectra (Figure S6) obtained for the single nanodevices highlight several new features. An absorption centered at $500 \mathrm{~nm}$ (Figure S6A: peak 1) increases in amplitude as nanocavity size is enlarged from 10 to $20 \mathrm{~nm}$. This enhanced absorption is well reproduced by simulation (Figure S6B: peak 1) and attributed to an increase in optical feedback (Figure S6C) due to change of refractive index ${ }^{29,30}$ within the porous nanostructure. In addition, a very weak absorption at $625 \mathrm{~nm}$ blue shifts by $40 \mathrm{~nm}$ and its amplitude increases by a factor of 2.0 as nanocavity size is increased by $10 \mathrm{~nm}$ (Figure S6A: peak 2). Simulation shows reasonable agreement with this trend, predicting a $30 \mathrm{~nm}$ blue shift and 2-fold increase in amplitude for the same nanocavity size change (Figure S6B: peak 2). Analysis of simulated absorption mode profiles ascribes the wavelength shift to mode-pulling (Figure S6C) towards the lower refractive index air-filled nanocavity. ${ }^{29,30} \mathrm{No}-$ tably, additional simulation results indicate that the spectral properties of these nanostructures can be significantly altered through subtle modification of the size and position of the internal nanocavities.

Nanoscale species may be delivered into the NW nanocavities. Immersion of the nanoporous structure in a $5 \mathrm{~nm} \mathrm{Au} \mathrm{col-}$ loid solution led to infiltration of $\mathrm{Au}$ nanoparticles into the nanocavities (Figure 4C). A planview TEM of the nanostructure following immersion shows $4 \mathrm{Au}$ nanoparticles trapped within a nanocavity (Figure 4C) that is encapsulated by intrinsic and $n$-type Si. This approach should be general for both metal and semiconductor nanoparticles as well as molecular dyes, thus opening up a new avenue for study of photosensitization and catalysis in unique electrically-addressable nanocavities. A nanostructure-catalyst framework presents a unique nanoporous scaffold within which to self-assemble catalysts; such a motif is appealing for the assembly of catalysts employed in energy conversion. ${ }^{31}$ In addition, we expect our approach can accomplish facet selective synthesis of III/V or II/VI semiconductor NW materials, and nanoporous NW structures of these materials, that will be interesting targets of future study.

\section{ASSOCIATED CONTENT}

Supporting Information. Detailed description of synthetic methods, sample preparation, and device fabrication; Supplementary Figures S1-S5; additional references. This material is available free of charge via the Internet at http://pubs.acs.org.

\section{AUTHOR INFORMATION}

\section{Corresponding Authors}

hgpark@korea.ac.kr; dnocera@fas.harvard.edu; cml@cmliris.harvard.edu

\section{Notes}

The authors declare no competing financial interest.

\section{ACKNOWLEDGMENTS}

T.J.K acknowledges a National Science Foundation Graduate Research Fellowship. C.M.L. acknowledges support of this research from a DOD NSSEFF (N00244-09-1-0078) Award. D.G.N. acknowledges support of NSF CCI (CHE-1305124). H.G.P. acknowledges a MSIP grant (2009-0081565) from the National Research Foundation of Korea.

\section{REFERENCES}

(1) Lu, W.; Lieber, C. M. Nat. Mater. 2007, 6, 841-850.

(2) Lu, W.; Lieber, C. M. J. Phys. D: Appl. Phys. 2006, 39, 387406.

(3) Xia, Y.; Yang, P.; Sun, Y.; Wu, Y.; Mayers, B.; Gates, B.; Yin, Y.; Kim, F.; Yan, H. Adv. Mater. 2003, 15, 353-389.

(4) Xiang, J.; Lu, W.; Hu, Y.; Wu, Y.; Yan, H.; Lieber, C. M. Nature 2006, 441, 489-493.

(5) Patolsky, F.; Timko, B. P.; Zheng, G.; Lieber, C. M. MRS Bull. 2007, 32, 142-149.

(6) Tian, B.; Cohen-Karni, T.; Qing, Q.; Duan, X.; Xie, P.; Lieber, C. M. Science 2010, 329, 830-834.

(7) Cohen-Karni, T.; Timko, B. P.; Weiss, L. E.; Lieber, C. M. Proc. Natl. Acad. Sci. USA 2009, 106, 7309-7313.

(8) Tian, B.; Kempa, T. J.; Lieber, C. M. Chem. Soc. Rev. 2009, 38, $16-24$.

(9) Kempa, T. J.; Cahoon, J. F.; Kim, S-K.; Day, R. W.; Bell, D. C.; Park, H-G.; Lieber, C. M. Proc. Natl. Acad. Sci. USA 2012, 109, $1407-1412$

(10) Kim, S-K.; Day, R. W.; Cahoon, J. F.; Kempa, T. J.; Song, K-Y.; Park, H-G.; Lieber, C. M. Nano. Lett. 2012, 12, 4971-4976.

(11) Cao, L.; White, J. S.; Park, J-S.; Schuller, J. A.; Clemens, B. M.; Brongersma, M. L. Nat. Mater. 2009, 8, 643-647.

(12) Kelzenberg, M. D.; Turner-Evans, D. B.; Kayes, B. M.; Filler, M. A.; Putnam, M. C.; Lewis, N. S.; Atwater, H. A. Nano. Lett. 2008, 8, 710-714.

(13) Kempa, T. J.; Tian, B.; Kim, D. R.; Hu, J.; Zheng, X.; Lieber, C. M. Nano. Lett. 2008, 8, 3456-3460.

(14) Tian, B.; Xie, P.; Kempa, T. J.; Bell, D. C.; Lieber, C. M. Nat. Nanotechnol. 2009, 4, 824-829.

(15) Qian, F.; Li, Y.; Gradecak, S.; Park, H-G.; Dong, Y.; Ding, Y.; Wang, Z. L.; Lieber, C. M. Nat. Mater. 2008, 7, 701-706.

(16) Caroff, P.; Dick, K. A.; Johansson, J.; Messing, M. E.; Deppert, K.; Samuelson, L. Nat. Nanotechnol. 2009, 4, 50-55.

(17) Wacaser, B. A.; Dick, K. A.; Johansson, J.; Borgstrom, M. T.; Deppert, K.; Samuelson, L. Adv. Mater. 2009, 21, 153-165.

(18) Schmidt, V.; Wittemann, J. V.; Gosele, U. Chem. Rev. 2010, 110, 361-388.

(19) Kodambaka, S.; Tersoff, J.; Reuter, M. C.; Ross, F. M. Science 2007, 316, 729-732.

(20) Eaglesham, D. J.; Cerullo, M. Phys. Rev. Lett. 1990, 64, 19431946.

(21) Kwon, S.; Chen, Z. C. Y.; Kim, J-H.; Xiang, J. Nano. Lett. 2012 12, 4757-4762

(22) Pan, L.; Lew, K-K.; Redwing, J. M.; Dickey, E. C. Nano. Lett. 2005, 5, 1081-1085.

(23) Ben-Ishai, M.; Patolsky, F. Adv. Mater. 2010, 22, 902-906.

(24) Tian, B.; Zheng, X.; Kempa, T. J.; Fang, Y.; Yu, N.; Yu, G.; Huang, J.; Lieber, C. M. Nature 2007, 449, 885-890.

(25) Cho, B.; Bareno, J.; Foo, Y. L.; Hong, S.; Spila, T.; Petrov, I.; Greene, J. E. J. Appl. Phys. 2008, 103, 123530:1-10.

(26) Wang, Z. L.; Kang, Z. C. Functional and smart materials: Structural evolution and structure analysis; Plenum Press: New York, 1998.

(27) Mullner, P.; Gao, H.; Ozkan, C. S. Philos. Mag. A 1997, 75, 925-938.

(28) Luth, H. Solid Surfaces, Interfaces, and Thin Films ed. 3; Springer: New York, 2001.

(29) Hecht, E. Optics ed. 4; Addison-Wesley: New York, 2002.

(30) Almeida, V. R.; Xu, Q.; Barrios, C. A.; Lipson, M. Opt. Lett. 2004, 29, 1209-1211.

(31) Surendranath, Y.; Lutterman, D. A.; Liu, Y.; Nocera, D. G. J. Am. Chem. Soc. 2012, 134, 6326-6336. 
Facet-selective growth of Ge on Si nanowires yields multi-component nanostructures and photonic devices.

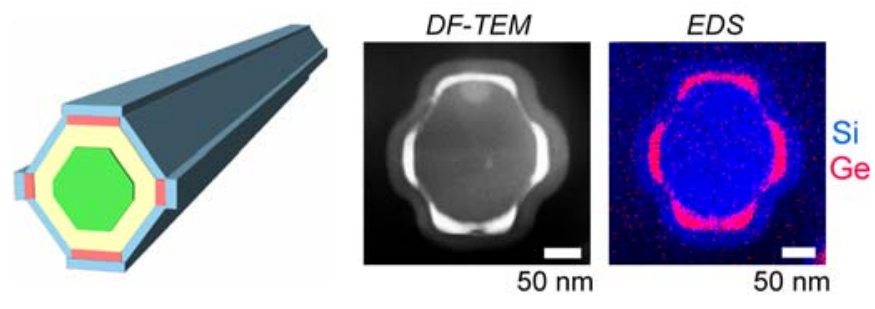




\title{
Supplementary Information
}

\section{Facet-Selective Growth on Nanowires Yields Multi-Component Nanostructures and Photonic Devices}

\author{
Thomas J. Kempa, ${ }^{\dagger}$ Sun-Kyung Kim, ${ }^{\dagger, \diamond}$ Robert W. Day, ${ }^{\dagger}$ Hong-Gyu Park, ${ }^{* \S}$ Daniel G. Nocera, ${ }^{* \dagger}$ \\ and Charles M. Lieber*十,‡ \\ tDepartment of Chemistry and Chemical Biology and $¥$ School of Engineering and Applied Sciences, \\ Harvard University, Cambridge, Massachusetts 02138. ^Department of Applied Physics, Kyung Hee \\ University, Gyeonggi-do, Republic of Korea. sDepartment of Physics, Korea University, Seoul 136-701, \\ Republic of Korea. \\ email: cml@cmliris.harvard.edu
}

Index

Page

Materials and Methods

S2-S5

Fig. S1 Role of template surface for facet selective growth of Ge

Fig. S2 Facet selective Si homoepitaxial growth

Fig. S3 TEM cross-section preparation and images

Fig. S4 EDS spectrum of nanostructure with embedded Ge regions

Fig. S5 TEM of Si/Ge/Si interface near $\{011\}$

S10

Fig. S6 EQE spectra of NW devices containing nanocavities 


\section{Materials and Methods}

\section{Nanowire Syntheses}

Si Nanowire (NW) Template. Au catalysts (100 nm diameter) were dispersed on poly-Llysine functionalized $600 \mathrm{~nm} \mathrm{SiO} 2$-on-Si wafers. Substrates were inserted into a home-built quartz-tube reactor and the system was evacuated to 2.8 mTorr base pressure. Crystalline intrinsic Si nanowire (NW) cores were grown at $460{ }^{\circ} \mathrm{C}$ and 40 Torr for $1 \mathrm{~h}$ with flow rates of 1 and 60 standard cubic centimeters per minute $(\mathrm{sccm})$ for silane $\left(\mathrm{SiH}_{4}\right)$, and hydrogen $\left(\mathrm{H}_{2}\right.$, Semiconductor Grade), respectively. A crystalline intrinsic Si shell was grown over these cores at $775{ }^{\circ} \mathrm{C}$ and 25 Torr for $30 \mathrm{~min}$ with flow rates of 0.15 and $60 \mathrm{sccm}$ for silane and hydrogen, respectively. ${ }^{1}$ A calibrated shell growth rate of $1.7 \mathrm{~nm} / \mathrm{min}$ was determined from independent studies of shell thickness vs. growth time.

Structure 1. Immediately following synthesis of the Si NW template, the reactor was purged to base pressure and re-pressurized to 4 Torr with a hydrogen flow rate of $20 \mathrm{sccm}$. This step was completed within 10 s. While pressurized, the reactor was cooled from 775 ${ }^{\circ} \mathrm{C}$ to $200{ }^{\circ} \mathrm{C}$ over $15 \mathrm{~min}$. Once the reactor cooled to be sure that the sample was below the final Ge growth temperature, the furnace lid was closed and its heater turned back on. The $\mathrm{Ge}$ growth temperature set point $\left(330^{\circ} \mathrm{C}\right)$ was reached within $3 \mathrm{~min}$. Facet selective synthesis of Ge was carried out at $330^{\circ} \mathrm{C}$ and 5.8 Torr for 5 min with flow rates of 10 and 20 sccm for germane $\left(\mathrm{GeH}_{4}, 10 \%\right.$ in $\left.\mathrm{H}_{2}\right)$ and hydrogen, respectively.

Structure 2. Immediately following synthesis of the Si NW template, the reactor was purged to base pressure and argon (Ar, Semiconductor Grade) introduced at a flow rate of $20 \mathrm{sccm}$. With argon continuously flowing, the reactor was cooled from $775{ }^{\circ} \mathrm{C}$ to $300{ }^{\circ} \mathrm{C}$ over $5 \mathrm{~min}$. Once the reactor cooled to be sure that the sample was below the final Ge growth temperature, the furnace lid was closed and its heater turned back on. The Ge growth temperature set point $\left(380^{\circ} \mathrm{C}\right)$ was reached within $3 \mathrm{~min}$. Facet selective synthesis of Ge was carried out at $380{ }^{\circ} \mathrm{C}$ and 10 Torr for 5 min with flow rates of 10,10 , and $20 \mathrm{sccm}$ for germane, phosphine $\left(\mathrm{PH}_{3}, 1000 \mathrm{ppm}\right.$ in $\left.\mathrm{H}_{2}\right)$ and argon, respectively.

Structure 3. Immediately following synthesis of the Si NW template, the reactor was purged to base pressure and cooled under vacuum from $775^{\circ} \mathrm{C}$ to $300{ }^{\circ} \mathrm{C}$ over $5 \mathrm{~min}$. Once the reactor cooled to be sure that the sample was below the final Ge growth temperature, the furnace lid was closed and its heater turned back on. The Ge growth temperature set point $\left(380^{\circ} \mathrm{C}\right)$ was reached within $3 \mathrm{~min}$. Synthesis of a conformal Ge shell was carried out at $380{ }^{\circ} \mathrm{C}$ and 4 Torr for 5 min with a flow rate of $5 \mathrm{sccm}$ for germane.

Structure in Fig. 3. Crystalline $p$-type Si NW cores were grown at $460{ }^{\circ} \mathrm{C}$ and 40 Torr for $1 \mathrm{~h}$ with flow rates of 1,5 and $60 \mathrm{sccm}$ for silane, diborane (100 ppm in $\mathrm{H}_{2}$ ) and hydrogen,

1 Kempa, T. J.; Cahoon, J. F.; Kim, S-K.; Day, R. W.; Bell, D. C.; Park, H-G.; Lieber, C. M. Proc. Natl. Acad. Sci. USA 2012, 109, 1407-1412. 
respectively. A crystalline $p$-type Si shell was grown over these cores at $775^{\circ} \mathrm{C}$ and 25 Torr for $5 \mathrm{~min}$ with flow rates of $0.15,1.5$ and $60 \mathrm{sccm}$ for silane, diborane and hydrogen, respectively. Next a crystalline intrinsic Si shell was grown over these cores at $775^{\circ} \mathrm{C}$ and 25 Torr for $15 \mathrm{~min}$ with flow rates of 0.15 and $60 \mathrm{sccm}$ for silane and hydrogen, respectively. Immediately following this synthesis, the reactor was purged to base pressure and re-pressurized to 4 Torr with a hydrogen flow rate of $20 \mathrm{sccm}$. While pressurized, the reactor was cooled from $775{ }^{\circ} \mathrm{C}$ to $200{ }^{\circ} \mathrm{C}$ over $15 \mathrm{~min}$. Once the reactor cooled, the furnace lid was closed and its heater turned back on. The Ge growth temperature set point $\left(330^{\circ} \mathrm{C}\right)$ was reached within $3 \mathrm{~min}$. Facet selective synthesis of Ge was carried out at $330^{\circ} \mathrm{C}$ and 5.8 Torr for $7 \mathrm{~min}$ with flow rates of 10 and $20 \mathrm{sccm}$ for germane and hydrogen, respectively. Next, the reactor was heated under vacuum to $550{ }^{\circ} \mathrm{C}$ and held at this temperature for 1 min. Synthesis of a conformal shell of $n$-type Si was carried out at $550{ }^{\circ} \mathrm{C}$ and 5 Torr for 1.5 min with flow rates of 2 and $10 \mathrm{sccm}$ for silane and phosphine.

Structure in Fig. S1. Immediately following synthesis of the Si NW template, the reactor was purged to base pressure and cooled under vacuum from $775{ }^{\circ} \mathrm{C}$ to $400{ }^{\circ} \mathrm{C}$ over $4 \mathrm{~min}$. Once the reactor cooled, the furnace lid was closed and its heater turned back on. The $\mathrm{Si}$ growth temperature set point $\left(450^{\circ} \mathrm{C}\right)$ was reached within $3 \mathrm{~min}$. Synthesis of a conformal amorphous Si layer was carried out at $450{ }^{\circ} \mathrm{C}$ and 5 Torr for 1 min with a flow rate of 2 sccm for silane. Next, the reactor was purged to base pressure, re-pressurized to 4 Torr with a hydrogen flow rate of $20 \mathrm{sccm}$, and cooled over $2 \mathrm{~min}$ to $200{ }^{\circ} \mathrm{C}$. Subsequent synthesis conditions were as for structure $\mathbf{1}$.

Structure in Fig. S2. Immediately following synthesis of the Si NW template, the reactor was purged to base pressure and hydrogen introduced at a flow rate of $60 \mathrm{sccm}$. With hydrogen continuously flowing, the reactor was cooled from $775{ }^{\circ} \mathrm{C}$ to $650{ }^{\circ} \mathrm{C}$ over $10 \mathrm{~min}$. Facet selective synthesis of $\mathrm{Si}$ was carried out at $650{ }^{\circ} \mathrm{C}$ and 25 Torr for 20 min with flow rates of 0.15 and $60 \mathrm{sccm}$ for silane and hydrogen, respectively.

\section{TEM and EDS Sample Preparation and Characterization}

Cross-sections for TEM studies were prepared by embedding NW structures in epoxy (EpoTek 353ND, Epoxy Technology). Samples were degassed to remove air bubbles and cured for $12 \mathrm{~h}$ at $30^{\circ} \mathrm{C}$ in a vacuum oven. A diamond knife (Ultra $35^{\circ}$, DiATOME) was used with a sectioning tool (Ultra Microtome, Leica) to cut $\sim 40-60 \mathrm{~nm}$ thick sections from the cured epoxy slugs. We note that the embedded nanowires have a distribution of orientation with respect to the growth substrate. During microtoming, this will lead to cross-sections that are cut off the nanowire growth axis, and thus yield a cross-section morphology that can vary from that expected for the ideal perpendicular section (Figure S3). The samples were transferred to lacey carbon grids for TEM analysis (JEOL 2100, JEOL Ltd.). An aberration corrected scanning TEM (cs-STEM, Libra 200 MC, Carl Zeiss NTS) equipped with twin EDS detectors and drift correction was used for acquisition of the EDS elemental map and 
spectrum shown in Figs. 3C and S3. These EDS data were acquired at $1024 \times 800$ resolution over $1 \mathrm{~h}$ using a $400 \mathrm{~ms}$ pixel dwell time and $1.2 \mathrm{~nm}$ spot size with beam energy of $200 \mathrm{kV}$. An SEM (Supra 55VP, Carl Zeiss NTS) equipped with EDS detector was used for acquisition of the EDS elemental maps shown in Fig. 1C. These EDS data were acquired at $512 \times 400$ resolution over 20 min using a $500 \mu$ s pixel dwell time with beam energy of $4 \mathrm{kV}$.

\section{NW Nanostructure Device Fabrication}

NW nanostructures were synthesized with 10 and $20 \mathrm{~nm}$ wide embedded Ge regions (Structure in Fig. 3.) and then covered with a $30 \mathrm{~nm}$ conformal layer of $\mathrm{SiO}_{2}$ using plasma enhanced chemical vapor deposition (PECVD). These nanostructures were shear transferred from their growth substrates to $\mathrm{Si}_{3} \mathrm{~N}_{4}{ }^{2}$ SU-8 2000.5 was spin-coated to a thickness of $500 \mathrm{~nm}$ over the device substrate, pre-baked $\left(95{ }^{\circ} \mathrm{C}\right)$, and electron beam lithography (EBL) was used to define SU-8 etch masks over a portion of the nanostructure. SU-8 was developed and cured for $10 \mathrm{~min}$ at $180^{\circ} \mathrm{C}$. Subsequently, etching to the $p$-type $\mathrm{Si}$ core was accomplished in the following order: $5 \mathrm{sec}$ in $\mathrm{BHF}$ (for removal of the outer $\mathrm{SiO}_{2}$ shell), $10 \mathrm{sec}$ in potassium hydroxide ( $\mathrm{KOH} 38 \mathrm{vol} . \%$ in water) at $60{ }^{\circ} \mathrm{C}, 10 \mathrm{~s}$ in hydrogen peroxide (30 vol.\% in water) at $60{ }^{\circ} \mathrm{C}$, and $12 \mathrm{sec}$ in potassium hydroxide at $60^{\circ} \mathrm{C}$. To selectively etch $\mathrm{Ge}$ from $^{3}$ the nanostructure, the device substrate was immersed in hydrogen peroxide at $60{ }^{\circ} \mathrm{C}$ for $30 \mathrm{~min}$. Lastly, EBL followed by thermal evaporation of 4 $\mathrm{nm}$ of Ti and $300 \mathrm{~nm}$ of Pd was performed to define ohmic contacts to the etched ( $p$-type) core and un-etched (n-type) shell. For the demonstration shown in Fig. 4C, a water solution containing $5 \mathrm{~nm} \mathrm{Au}$ nanoparticles (Ted Pella, Inc.) was dropped onto the surface of an epoxy plug and allowed to evaporate. This plug was previously embedded with the Geetched nanowire structures (Fig. 4B) whose ends were exposed at its surface. Longitudinal TEM cross-sections $\sim 100 \mathrm{~nm}$ thick were prepared by microtoming this slug at a $70^{\circ}$ angle.

\section{Device EQE spectra}

Polarization-resolved photocurrent spectra were obtained on a home-built optical setup1 utilizing a standard solar simulator ( $150 \mathrm{~W}$, Newport Oriel) with AM 1.5G filter as illumination source, a spectrometer (SpectraPro 300i, Acton Research) with $1200 \mathrm{~g} / \mathrm{mm}$ grating and blaze angle of $500 \mathrm{~nm}$, and an uncoated Glan-Thompson calcite polarizer (10GT04, Newport). Illumination power was measured using a power meter and lowpower Si photodetector (1918-C and 918D-UV-OD3, Newport). Power spectra were acquired from 300 to $900 \mathrm{~nm}$ in $5 \mathrm{~nm}$ increments through 1.0, 1.3, and $2.0 \mathrm{~mm}$ diameter circular apertures to verify uniformity and accuracy of the power density used to calculate absolute EQE values. Nanostructure device photocurrents for transverse-electric (TE) and

2 Javey, A.; Nam, S. W.; Friedman, R. S.; Yan, H.; Lieber, C. M. Nano Lett. 2007, 7, 773-777.

3 Williams, K. R.; Gupta, K.; Wasilik, M. J. Microelectromech. S. 2003, 12, 761-778. 
transverse-magnetic (TM) polarizations were measured from 300 to $900 \mathrm{~nm}$ in $5 \mathrm{~nm}$ increments using a semiconductor parameter analyzer (4156C, Agilent Technologies). Absolute EQE spectra were calculated using the wavelength-dependent photocurrent data collected as described above and the projected area of the nanostructures, which was measured by SEM. The projected area of the nanostructure was taken to be the exposed area of the un-etched nanostructure when viewed perpendicular to the substrate plane and did not include the area covered by the $300 \mathrm{~nm}$ thick metal contacts.

\section{FDTD calculations}

The absorption cross section of the simulated nanostructures under illumination by a normally incident plane wave was obtained by integrating $J$.E at each grid point, where $J$ and $\mathrm{E}$ are the polarization current density and electric field, respectively. The absorption cross section was integrated over one optical period, and the wavelength of incident light was scanned from $280-1000 \mathrm{~nm}$ in $5 \mathrm{~nm}$ increments. The absorption efficiency is the ratio of the nanostructure absorption cross section to its physical cross section. Lastly, EQE is calculated by multiplying the absorption efficiency by internal quantum efficiency (IQE), where IQE was assumed to be unity. The nanostructure was simulated as a volume element with hexagonal cross-section and a spatial resolution of $5 / \sqrt{3}, 5$, and $5 \mathrm{~nm}$ for $\mathrm{x}, \mathrm{y}$ and $\mathrm{z}$, respectively, where y lies along the nanostructure axis and $\mathrm{z}$ lies along the propagation direction of the incident plane wave. The simulated nanostructures had diameters of 230 $\mathrm{nm}$, with 0,10 , or $20 \mathrm{~nm}$ wide air-filled cavities positioned $20 \mathrm{~nm}$ from the Si surface. All simulations included the $\mathrm{Si}_{3} \mathrm{~N}_{4}$ device substrate and conformal $\mathrm{SiO}_{2}$ layer used in the experiment. Periodic boundary conditions were applied along the axis of the nanostructure. To ensure that a single nanostructure experiences an infinite plane wave, we implemented the total-field scattered-field (TFSF) method. Without this method, a single nanostructure would be simulated as a periodic array of nanostructures along the $\mathrm{x}$ axis. The measured refractive index and extinction coefficient of single crystal silicon ${ }^{4}$ over the wavelength range, $280-1000 \mathrm{~nm}$, was incorporated into the FDTD simulation.

4 Lide, D. R. CRC Handbook of Chemistry and Physics: A Ready-reference Book of Chemical and Physical Data; CRC Press: Boca Raton, 2008. 
(A)

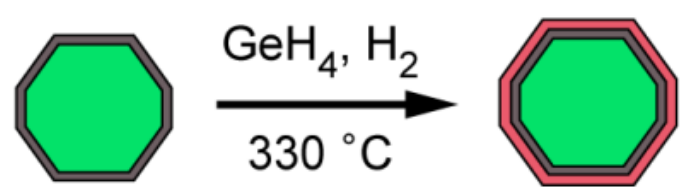

(B)

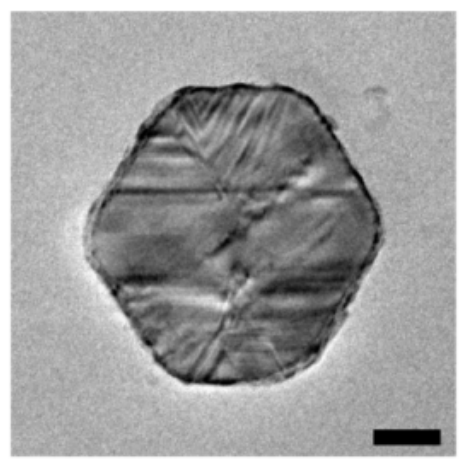

(C)

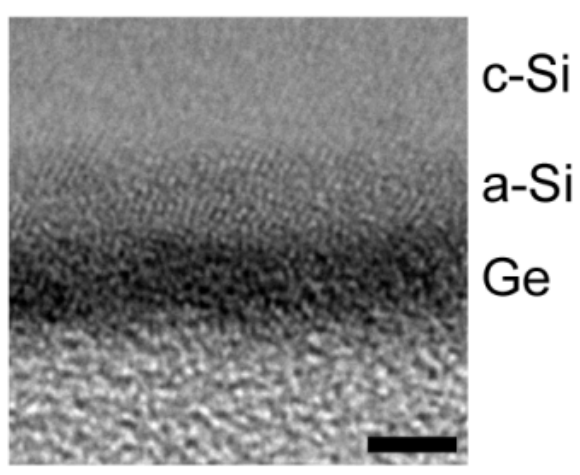

Figure S1. Role of template surface for facet selective growth of Ge. (A) Schematic of synthesis conditions which are identical to those for structure $\mathbf{1}$. The Si template (green) has been covered with an amorphous Si shell (gray). (B) Bright-field TEM of a $40 \mathrm{~nm}$ thick cross-section of the nanostructure grown as outlined in (A). Image is oriented with $\{111\}$ surfaces on top and bottom. Scale bar, $50 \mathrm{~nm}$. (C) High-resolution TEM near $\{111\}$ surface identifying the intentionally deposited amorphous Si shell and a thin conformal Ge shell. Scale bar, $3 \mathrm{~nm}$. 
(A)

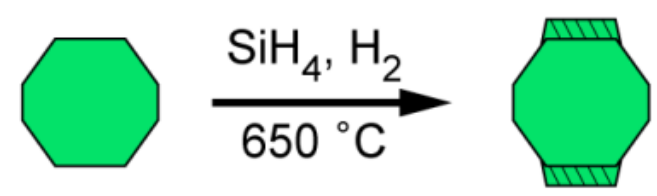

(B)

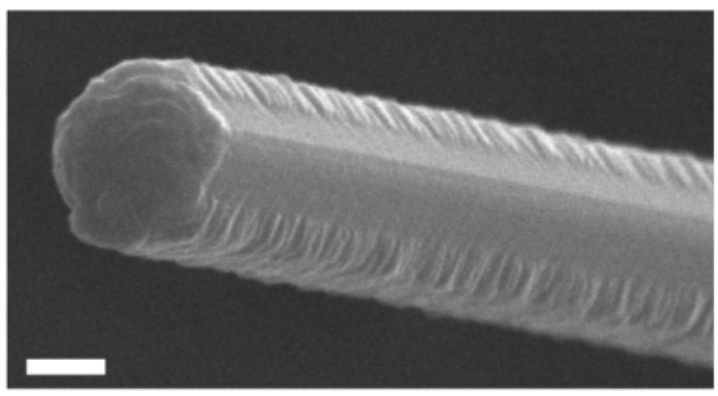

(C)

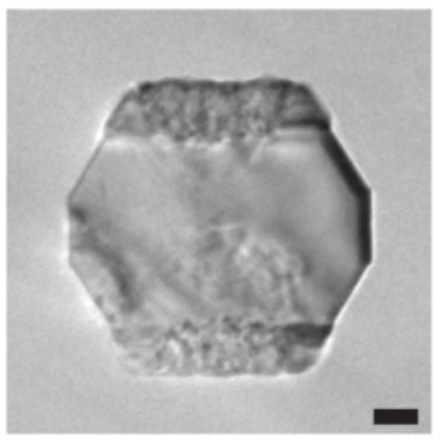

Figure S2. Facet selective Si homoepitaxial growth. (A) Schematic outlining synthesis conditions for facet-selective growth of $\mathrm{Si}$ on a Si template (green). (B) SEM of the nanostructure grown as outlined in (A). Image is oriented with $\{111\}$ surfaces on top and bottom. Scale bar, $200 \mathrm{~nm}$. (C) Bright-field TEM of nanostructure cross-section with $\{111\}$ surfaces oriented on top and bottom of image. Scale bar, $50 \mathrm{~nm}$. Facet-selective $\mathrm{Si}$ deposition is clearly visible on the $\{111\}$ surfaces. By comparing the dimensions and facet lengths of the nanostructure against these same values for the template we confirmed that no Si grew on the $\{113\}$ and $\{011\}$ surfaces. 


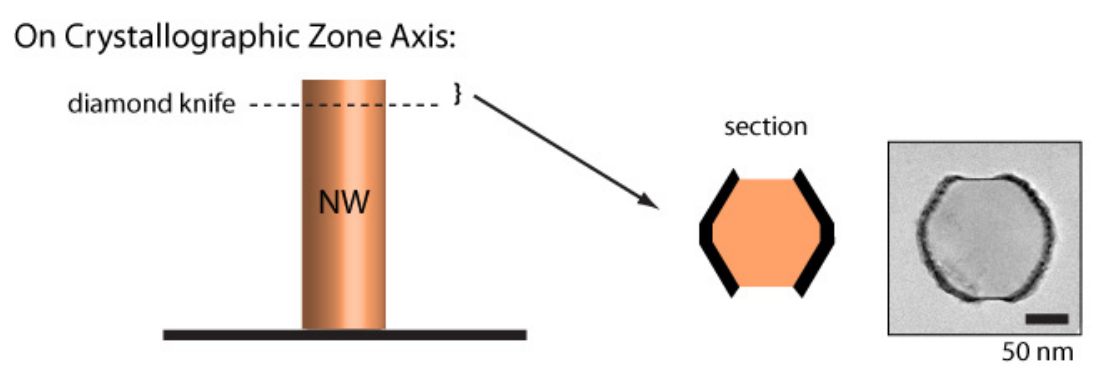

Off Crystallographic Zone Axis:

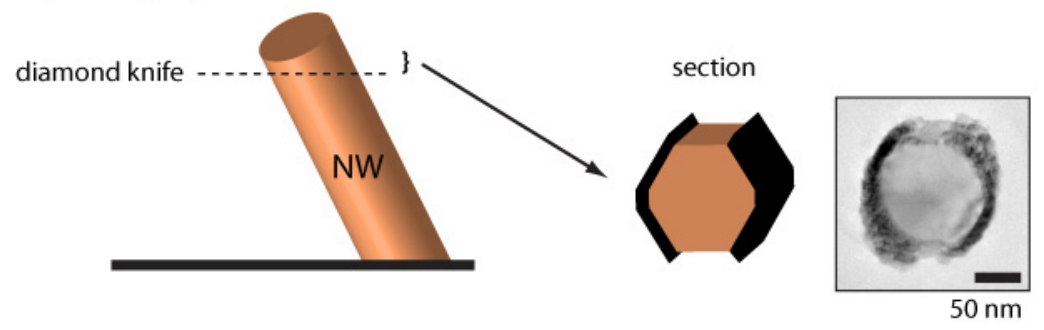

Figure S3. TEM cross-section preparation and images. As grown NWs have a range of orientations with respect to the growth substrate, and the sectioning plane of the diamond knife is parallel to the growth substrate for TEM cross-section sample preparation. In the ideal case, the diamond knife produces a section orthogonal to the NW's growth axis producing an undistorted axial slice when viewed in the TEM. In most cases, the diamond knife intercepts the NW at an angle off the nanowire axis thereby producing a distorted axial slice as observed in many TEM images. The upper and lower TEM images in the figure show ca. undistorted perpendicular and distorted off-axis cases, respectively. 


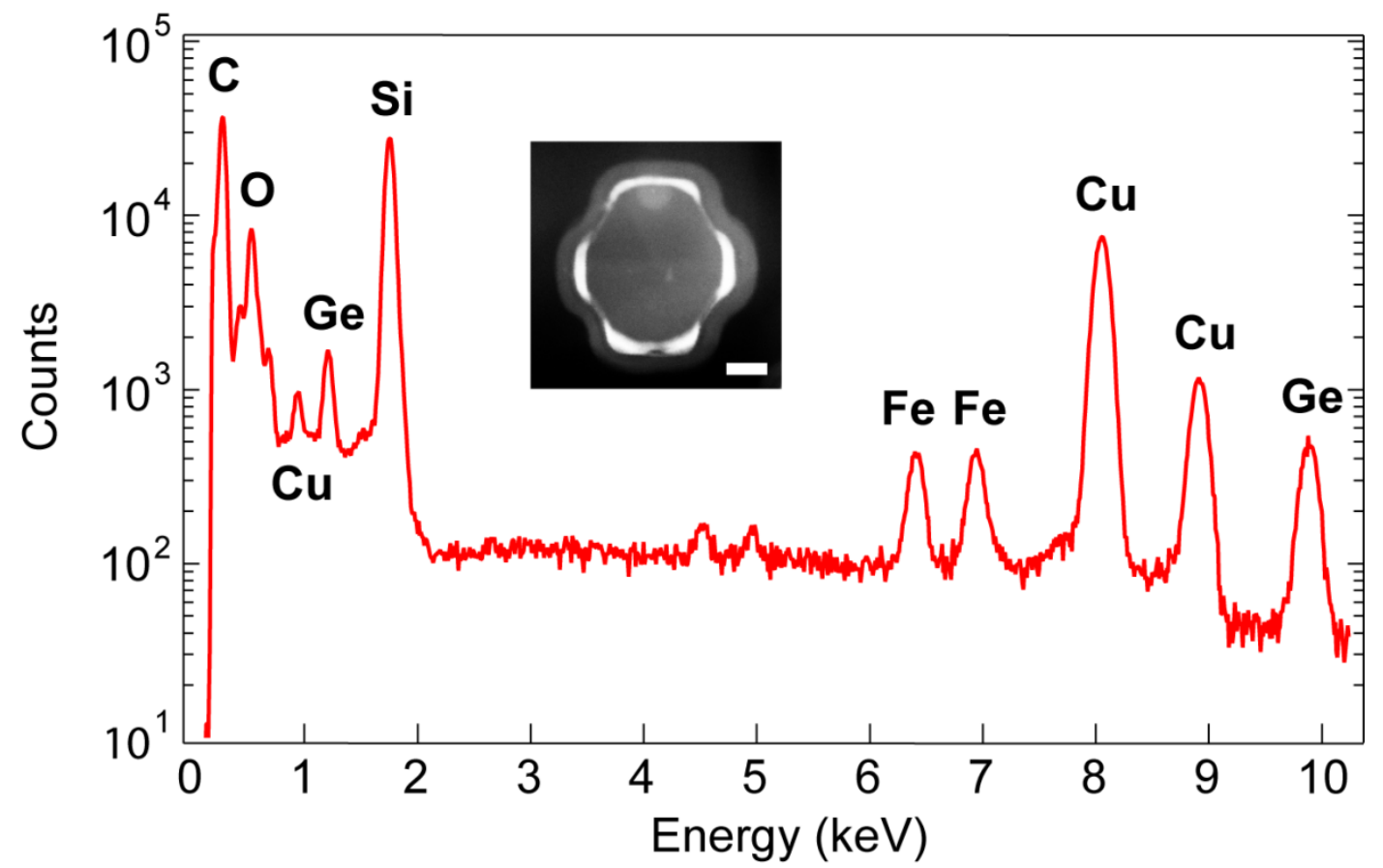

Figure S4. EDS spectrum of nanostructure with embedded Ge regions. EDS spectrum of a $40 \mathrm{~nm}$ thick cross-section (inset) of the nanostructure with Ge embedded within Si $p$ - $n$ junction. Ge L $\beta(1.21 \mathrm{keV}), \mathrm{Ge} \mathrm{K} \alpha(9.88 \mathrm{keV})$, and $\mathrm{Si} \mathrm{K} \alpha(1.76 \mathrm{keV})$ lines are clearly discernable. Inset: dark-field TEM of the cross-section used to acquire the EDS spectrum. Scale bar, $50 \mathrm{~nm}$. 


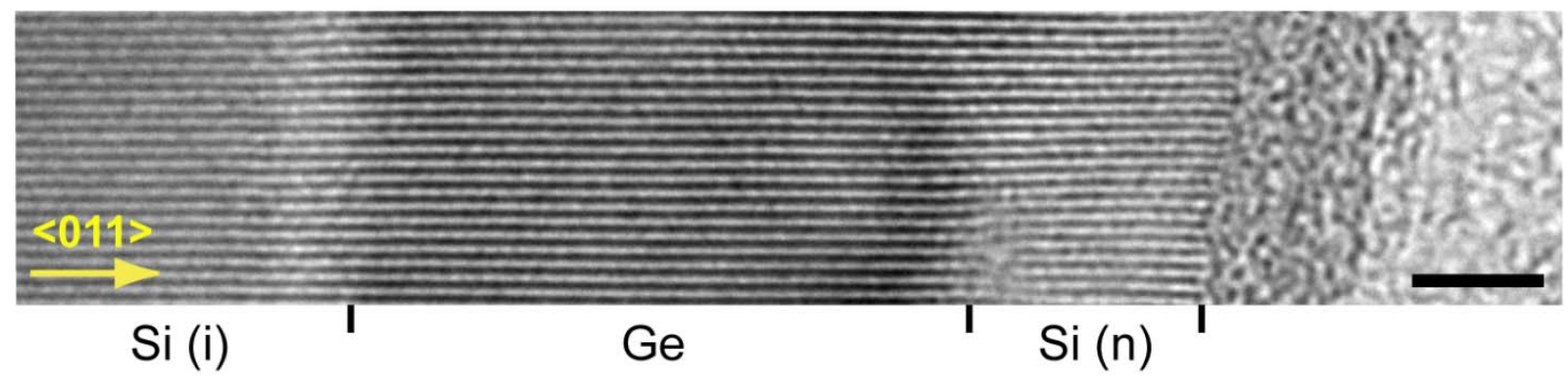

Figure S5. TEM of Si/Ge/Si interface near $\mathbf{0 1 1}\}$. High-resolution TEM of the intrinsic $\mathrm{Si} / \mathrm{Ge} / n$-Si region near the $\{011\}$ interface within the nanostructure. The $\{011\}$ plane lies parallel to the $y$-axis of the image. Scale bar, $3 \mathrm{~nm}$. 

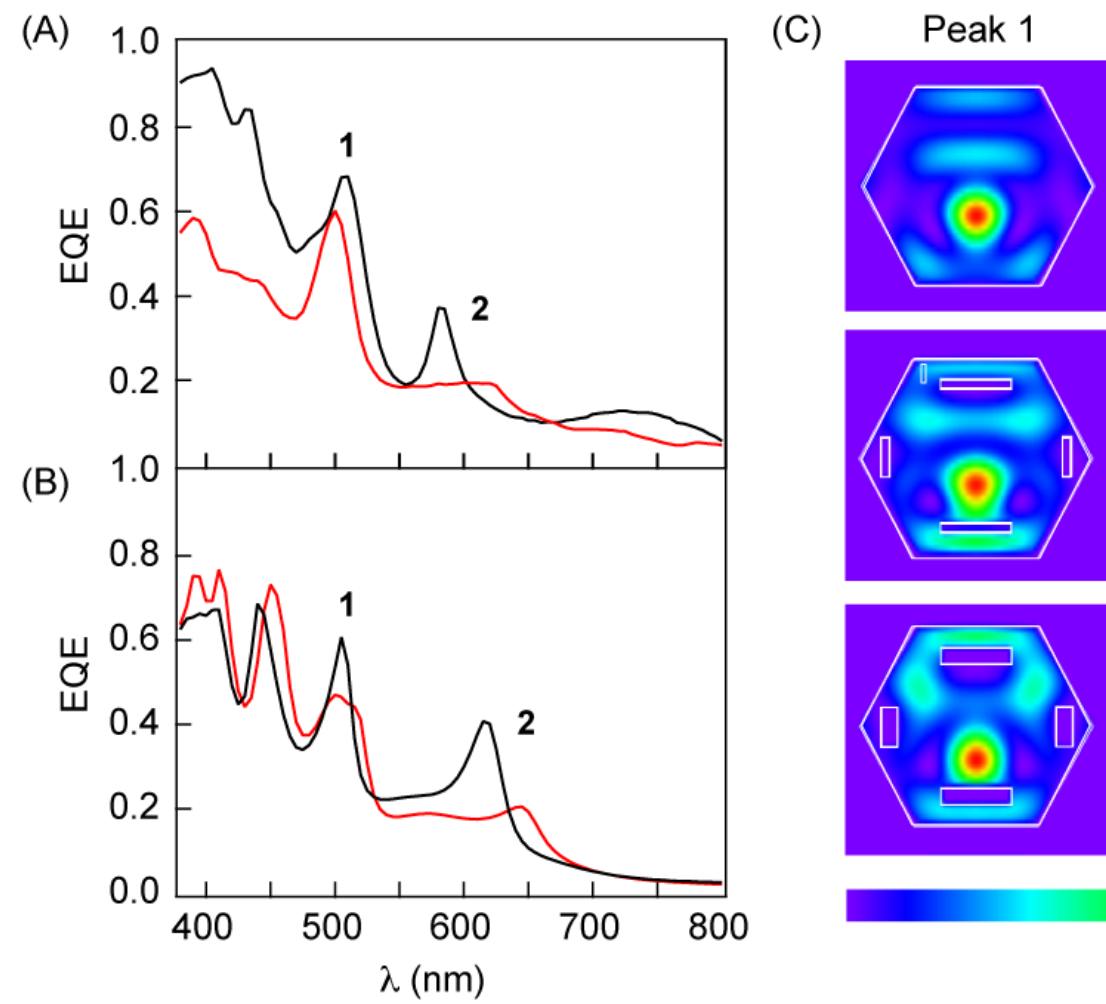

Peak 2
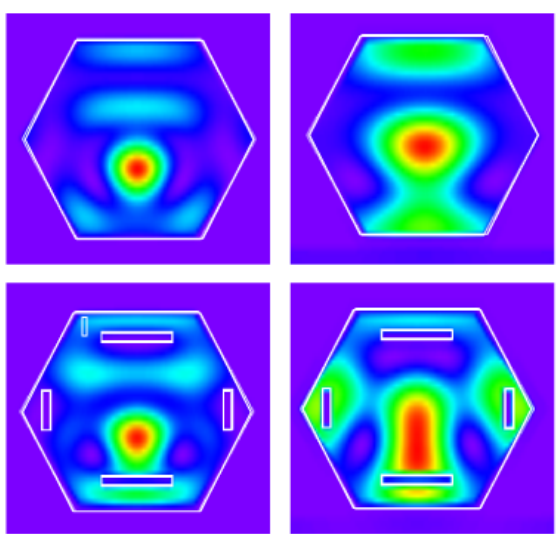

$\mathrm{w}=0 \mathrm{~nm}$
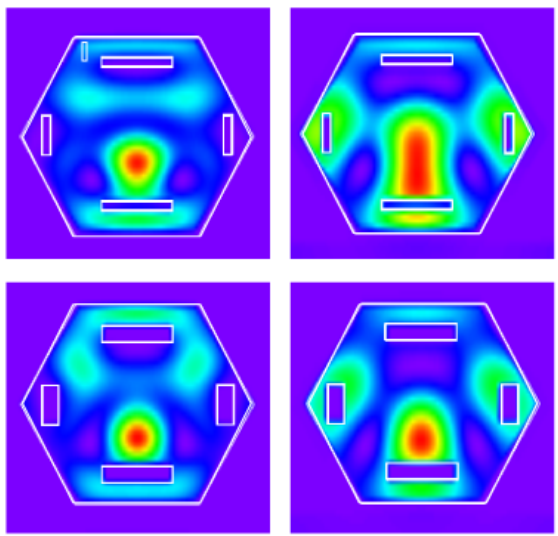

$\mathrm{w}=10 \mathrm{~nm}$

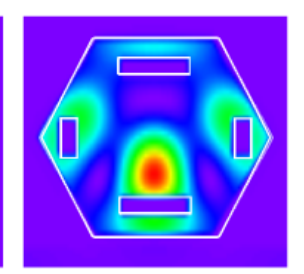

$\mathrm{w}=20 \mathrm{~nm}$

Figure S6. EQE spectra of NW devices containing nanocavities. (A) Experimental and simulated (B) absolute EQE spectra for the transverse-magnetic (TM) electric field polarization. Red and black lines correspond to nanostructures with 10 and $20 \mathrm{~nm}$ wide nanocavities, respectively. FDTD simulates nanostructures with hexagonal cross-sections, $230 \mathrm{~nm}$ diameters, and 10 and $20 \mathrm{~nm}$ wide cavities positioned $20 \mathrm{~nm}$ away from the outer Si surface of the nanostructure. SEM analyses of the devices used to obtain data in (A) show they have diameters of 224 and $236 \mathrm{~nm}$. These values are within $2.6 \%$ of the simulated diameter. (C) Simulated absorption mode profiles for peak $1(505 \mathrm{~nm})$ and peak 2 (685, 645 , and $615 \mathrm{~nm}$ ) for cavity sizes of 0,10 , and $20 \mathrm{~nm}$. 